\title{
Normal Electrooculography in Best Disease and Autosomal Recessive Bestrophinopathy
}

\section{Abbreviated Title: Normal EOG in BD and ARB}

Kamron N Khan, PhD, FRCOphth ${ }^{1-3}$

Farrah Islam, FCPS, FRCS. ${ }^{2}$

Graham E Holder, $\mathrm{PhD}^{1,2}$

Anthony Robson, $\mathrm{PhD}^{1,2}$

Andrew R Webster, MD(Res) , FRCOphth ${ }^{1,2}$

Anthony T Moore, FRCS, FRCOPhth ${ }^{1,2,4}$

Michel Michaelides, MD(Res), FRCOphth ${ }^{1,2}$

1. University College London Institute of Ophthalmology, University College London, London, UK.

2. Medical Retina Service, Moorfields Eye Hospital, London, UK.

3. Department of Ophthalmology, Leeds Institute of Molecular Medicine, St James' University Hospital, Beckett St, Leeds, UK.

4. Ophthalmology Department, University of California San Francisco Medical School, San Francisco, California, USA.

Corresponding authors: Kamron Khan and Michel Michaelides at address 1 above. Email:

medknk@leeds.ac.uk and michel.michaelides@ucl.ac.uk

Grants/ Financial Disclosure: National Institute for Health Research Biomedical Research Centre at Moorfields Eye Hospital National Health Service Foundation Trust and UCL Institute of Ophthalmology (UK; KNK, ARW, ATM, MM), Fight For Sight (UK; ARW, MM, OAM), Moorfields Eye Hospital Special Trustees (UK; MM), the Foundation Fighting Blindness (FFB, USA; ARW, ATM, MM), Retinitis Pigmentosa Fighting Blindness (UK; ARW, ATM, MM), and the Wellcome Trust (099173/Z/12/Z; ARW, MM). Michel Michaelides is supported by an FFB Career Development Award. This research has been funded/supported by the National Institute for Health Research Rare Diseases Translational Research Collaboration (NIHR RD-TRC). The views expressed are those of the author(s) and not necessarily those of the NHS, the NIHR or the Department of Health." 
Proprietary Interest : None

CONFLICT OF INTEREST: No conflicting relationship exists for any of the authors.

KEY WORDS

Autosomal Recessive Best Retinopathy.

Best related Retinopathy.

Electrooculography.

Retina.

Retinal dystrophy.

SUMMARY STATEMENT

The Bestrophinopathies are a group of inherited eye disorders that arise from either dominant or recessive mutations in the BEST1 gene. Electrooculography is invaluable in the diagnosis of Best disease (BD) and autosomal recessive bestrophinopathy (ARB), as a reduced Arden ratio is a highly penetrant feature of disease. We demonstrate that EOG phenotype in BD and ARB is more variable than currently appreciated. 


\section{Normal Electrooculography in Best Disease and Autosomal Recessive Bestrophinopathy}

\section{Abstract}

Purpose: To evaluate the electrooculogram (EOG) in a large series of patients with Best disease (BD) and autosomal recessive Bestrophinopathy (ARB).

Methods: A retrospective review of consecutive cases at Moorfields Eye Hospital, London, United Kingdom. Patients with BD or ARB who, after electrophysiological testing, had a normal or atypical EOG light rise were identified. Main outcome measure was EOG amplitude, clinical phenotype and genotype.

Results: 113 patients were identified with likely disease-causing sequence variants in BEST1 (99 BD, 14 ARB). EOGs had been performed in 75 patients. 20 patients (27\%) had no detectable light rise (Arden ratio of 100\%) and $49(65 \%)$ had Arden ratios of between $100-165 \%$. Six patients (8\%) were found to have an EOG light rise of $>165 \%$. No cases demonstrated significant interocular asymmetry in EOG amplitude.

Conclusions: The current work provides significant clinical evidence that the EOG phenotype in BD and ARB is more variable than currently appreciated. As a normal EOG may occur in the presence of a classical fundus appearance, the consequences of BEST1 mutation may be independently expressed, possibly mediated via differential effects on intracellular calcium homeostasis. 


\section{Normal Electrooculography in Best Disease and Autosomal Recessive Bestrophinopathy}

\section{Introduction}

The Bestrophinopathies are a group of inherited eye disorders that arise from either dominant or recessive mutations in the BEST1 gene. The clinical spectrum of disorders include (i) diseases predominantly affecting the macula - Best disease (BD) and Adult Vitelliform Macular Dystrophy (AVMD); (ii) generalised retinal involvement - Autosomal Dominant Vitreoretinochoroidopathy (ADVIRC) and Retinitis Pigmentosa (RP); and (iii) diseases with retinal and anterior segment involvement - autosomal recessive Bestrophinopathy (ARB) and Microcornea, Rod-cone dystrophy, Cataract and posterior Staphyloma (MRCS). ${ }^{1-7}$ In addition to the phenotypic heterogeneity some of the bestrophinopathies display significant variation in penetrance of the clinical phenotype. ${ }^{8}$ However, one endophenotype, the absent or reduced electrooculogram (EOG) light rise, is reported to be almost fully penetrant. ${ }^{9}$ This electrophysiological parameter arises as the cornea is positively charged relative to the negative retina thus creating a dipole through the eye that can be measured with surface electrodes. The light rise reflects a progressive depolarisation of the basal membrane of the retinal pigment epithelium (RPE) in response to a change from scotopic to photopic adaptation. Although the underlying molecular basis for the change in EOG with illumination is not known, it is almost always reduced in patients with BEST1 mutations. The present report investigates the EOG findings in a large cohort of patients with BD and ARB.

\section{Methods}

A retrospective review of the genetic database at Moorfields Eye Hospital, London, United Kingdom was undertaken to identify all patients with either bi-allelic or heterozygous mutations in BEST1 and a compatible clinical phenotype. Within this group only those who had undergone electrophysiological testing at our own institution were investigated further. Patients with a normal or atypical EOG light rise were then identified. 
The EOG testing was performed to be consistent with ISCEV standards. ${ }^{10}$ Normative EOG data were obtained by testing 30 individuals aged between 30 and 60 years of age with no known ocular abnormalities. One eye from each normal subject was randomly selected and used to establish the normative data. In brief, EOG testing was performed with surface electrodes placed at the medial and lateral canthi of each eye. Thirty degree eye movements were recorded for 10 seconds of each minute during 15 minutes of dark adaptation, followed by a further 10-15 minutes in full-field (Ganzfeld) light adaptation $\left(100 \mathrm{~cd} / \mathrm{m}^{2}\right)$. The amplitudes of the light peak and dark trough were directly measured and the light peak:dark trough ratio expressed as a percentage. Pattern electroretinograms (PERGs) and full-field ERGs (FFERGs) were used to assess macular function and generalised retinal function respectively. ${ }^{11,12}$ All clinical electrophysiological assessments were analysed by one of two consultant electrophysiologists (GH or AR) masked to the molecular diagnosis.

A clinical diagnosis was made based on the fundoscopic appearance together with findings on optical coherence tomography (OCT) and fundus autofluorescence (FAF) (excitation wavelength 488 nm; Spectralis, Heidelberg Engineering, Heidelberg, Germany). A genetic diagnosis was determined by DNA sequencing (Manchester Regional Genetics Laboratory). Genomic DNA was extracted from peripheral blood (Qiagen Blood DNA extraction kit, Qiagen, Crawley, UK) and used as the template for bidirectional Sanger sequencing analysis of the entire coding regions of BEST1, including the splice donor and acceptor sites.

\section{Results}

The EOG Arden ratios in the normal subjects ranged from $180 \%$ to $435 \%$ (median $267 \%$, SD $50 \%$ $\mathrm{n}=30$ ). For this study we considered an Arden ratio of less than $165 \%$ to be abnormal (2SD below the mean). 
A total of 113 patients were identified with likely disease-causing sequence variants in BEST1 (99 BD, 14 ARB). Electrophysiological testing had been performed in 75 patients. Three groups were identified. 20 patients (27\%) had no detectable light rise (Arden ratio of 100\%), whilst 49 cases (65\%) had Arden ratios of between $100-165 \%$. Six patients (8\%) were found to have an EOG light rise of $>165 \%$ and will be described in detail below. No cases were identified with sufficient asymmetry in Arden ratios to cause eyes of the same patient to be categorised differently.

Patient 1 was diagnosed with BD aged 8 based on bilateral typical macular vitelliform lesions. He later presented with reduced vision in his right eye due to macular scarring from a presumed occult choroidal neovascular membrane and underwent EOG testing aged 39. Arden ratios were $220 \%$ right and $180 \%$ left. FFERGs were normal and symmetrical, indicating no evidence of generalised retinal dysfunction. PERG P50 component was mildly subnormal on the right consistent with mild right macular dysfunction. The diagnosis of $\mathrm{BD}$ was thereby questioned and $P R P H 2$ gene screening was initiated but no pathogenic variants were identified. BEST1 screening revealed a heterozygous missense amino-acid substitution (p.Arg25Trp) previously associated with BD. ${ }^{13}$

Patient 2 was diagnosed with BD as a teenager when her vision deteriorated in both eyes. The diagnosis was questioned when she was seen between the ages of 28-36 due to an atypical macular appearance. OCT imaging demonstrated focal choroidal excavation alongside subretinal deposit and fluid. The FFERG was normal, but the PERG P50 component was reduced, left more than right in keeping with macular dysfunction. Eye movement artefacts disrupted accurate grading of the EOG but an adequate light rise and dark trough were noted, consequently this patient was reported to have a normal EOG and included in this cohort. Screening BEST1 identified the variant p.Lys30Asn, predicted to be damaging as this residue is highly conserved (PolyPhen2 score 1.0). Although the EOG was reported as normal, accurate quantification of the Arden ratio had not been possible. As part of this study the EOG was then repeated 12 months later, this time a reduced light rise with an Arden index of $120 \%$ in each eye was recorded, consistent with the diagnosis of BD. 
Patient 3 was diagnosed in his youth with a macular dystrophy that resembled BD. He was part of a consanguineous pedigree with a similarly affected brother living abroad. When he was examined aged 41 the right macula was found to be atrophic and the left showed subfoveal fluid and subretinal deposit (Figure 1). The EOG light rise to dark trough ratio was $175 \%$ on the right and $260 \%$ on the left. The PERG P50 component was bilaterally subnormal in keeping with macular dysfunction. FFERGs were normal. BEST1 gene screening identified the homozygous variant p.Arg47Cys, previously shown to cause ARB, also with a vitelliform rather than multifocal phenotype. ${ }^{14,15}$

Patient 4 was diagnosed with BD aged 9 based on a vitelliform maculopathy and dominant family history. The EOG light rise to dark trough ratio was $180 \%$ bilaterally. There was PERG evidence of mild left macular dysfunction. FFERGs were normal bilaterally. Screening of BEST1 revealed a previously reported heterozygous mutation p.Tyr85His. ${ }^{16}$

Patient 5 was diagnosed in childhood with BD and was examined again at the age of 33 . She had a small vitelliform lesion in her right eye and a petaloid arrangement of subretinal deposit in her left eye in keeping with a reticular pattern dystrophy-like phenotype. No similarly affected family members were known. The FFERG and PERG were normal and her EOG light rise to dark trough ratio was $175 \%$ on the right and $180 \%$ on the left. DNA sequencing identified a BEST1 variant (p.Ala243Val) already known to cause disease BD. ${ }^{3}$

Patient 6 had a history of poor vision from the age of 12 and was found to be hyperopic at the age of 20 years old. His left eye was amblyopic. Both his parents had good vision into their seventh decade. By the age of 37 his vision had declined to hand movement in the right eye and 1/60 in the left eye. He was diagnosed with $\mathrm{BD}$ after retinal examination revealed a vitelliform maculopathy in his right eye and well defined atrophy in the left (Figure 2). The FFERG was normal, but the PERG was reduced. The EOG light rise was recorded at $200 \%$ in each eye. Screening the genes $P R P H 2$ and BEST1 identified p.Leu567Phe in BEST1 as the only likely pathological variant. This variant has been 
previously reported as a possible cause of late adulthood onset retinal dystrophy and theoretically is sufficiently rare to cause BD, (0/61730511, Exome Aggregation Consortium (ExAC) database. ${ }^{17,18}$ As it remains questionable whether this variant accounts for the maculopathy present in Patient 6, whole genome sequencing is being employed to elucidate the genetic cause.

\section{Discussion}

In this cohort of patients with molecularly proven BD or ARB, four individuals (5.4\%) were found to have a normal, or greater EOG light rise than usually seen in the bestrophinopathies. This was a higher number than expected, as although BEST1 mutations are associated with varied expression of the fundus phenotype, it is considered a rarity that the EOG light rise remains unaffected, with only a few individual cases reported. ${ }^{3,13-15,19-24}$ The present findings, when combined with published data, demonstrate that of the 269 unique BEST1 mutations thus far collated, at least $3.3 \%$ have now been associated with a greater than expected EOG amplitude (Table 1). ${ }^{25}$

In collecting these data we are able to highlight two potential reasons whereby erroneous conclusions may be made when interpreting the results of either electrophysiological or genetic testing. Firstly, inaccurate saccadic eye movements in Patient 2 produced the illusion of a falsely high Arden ratio. This was noticed by careful review of the traces and corrected by retesting. Secondly, pathogenicity has probably been wrongly assigned to the variant p.Leu567Phe identified in Patient 6; given that it has only been seen twice before (and only in the context of age-related macular degeneration), is not a highly conserved residue, and when modelled in vitro does not alter chloride conductance or channel permeability to anions. ${ }^{26,27}$ This case highlights the increasing challenge that is faced in definitively ascribing disease-causation to identified sequence variants, especially for missense mutations.

Why a subgroup of patients retain a light rise is currently unknown. Theoretically it is possible that they represent a group who, in the absence of a mutation, would have recorded an unusually high 
light rise. Therefore, whilst the effects of mutation result in a reduction of amplitude, this only serves to bring it to the lower end of the normal range. Data derived from testing normal individuals herein shows a more than two fold difference between the lowest and highest Arden ratios (180435\%) and the authors do have experience of at least one normal subject with a light rise of more than $500 \%$ (unpublished data). Given these findings, it is likely that genetic modifiers exist which may influence the EOG amplitude, either via effects on BEST1 itself or interacting proteins. One hypothesis, that may also underpin the variation in fundus expression, proposes that a normal EOG may result from relatively higher expression of the wild-type compared to mutant allele. Whilst allelic imbalance is a plausible explanation for dominant disease, it would be harder to argue in cases of recessive inheritance, where the ability to maintain a light rise must depend upon presence of some retained BEST1 function. Recessive alleles are thought to be functionally null, however this may not always be the case. The p.Arg47Cys mutation (patient 3 in our series) has now occurred with relatively mild EOG on three independent occasions and in different ethnic populations. ${ }^{14,15} \mathrm{An}$ additional atypical finding in these cases is the presence of unifocal disease, as recessive disease is usually more widespread. It is possible that these alleles are hypomorphic and retain sufficient function to support anion transport across the basolateral surface of the RPE thus maintaining an EOG light rise, and at the same time have enough function to limit the disease to the macula. The same mechanism may also be proposed for the recessive c.1100+1G>A allele, suggesting that aberrant spicing does affect all transcripts. ${ }^{24}$

Interestingly, all the mutations that have been associated with a normal EOG have also been described in individuals with a poor or absent light rise on EOG. This variability has been observed in members of the same family, again suggesting the influence of genetic or environmental modifiers. ${ }^{23}$ Some variants however are overrepresented within the normal EOG group, particularly the p.Ala243Val mutation. Out of 42 unique heterozygous mutations identified in this cohort, p.Ala243Val was the second most commonly reported $(n=6)$, the most prevalent being p.Arg218Cys $(n=10)$. Whilst this could suggest that the association with a normal EOG is a chance finding, others 
have also reported this genotype with a normal Arden ratio (Table 1). If chance alone were acting one would expect that the most commonly encountered mutation (p.Arg218Cys) would be most often associated with a normal Arden ratio. This has only been described once before and in our series this genotype always had an abnormal EOG.$^{19}$ The ability to generate a light rise therefore may in some cases be strongly influenced by the genotype (p.Arg47Cys, p.Ala234Val) whilst other factors, yet to be determined, are responsible for the remainder.

Whilst the molecular basis supporting the light rise of the EOG has yet to be established, our understanding of conditions that may alter it has advanced. The polarised RPE is thought to generate and maintain a potential difference across its surface, and this can be affected in both health and disease. ${ }^{28-30}$ In the presence of light, the conduction of anions across the basolateral RPE membrane is thought to increase, resulting in depolarisation and a rise in the recorded potential difference. This process may be pharmacologically manipulated, as lamotrigine and acetazolamide have both been shown to reduce the amplitude of the EOG light rise. ${ }^{31-33}$ Additionally, the stimulus for the light rise does not need to be photic, as alcohol (supplied by the choroidal circulation) simulates this phenomenon. ${ }^{30}$ In the disease state, the RPE may either not be able to maintain its polar properties due to disorganisation of tight junctions, or may not be able to pump anions efficiently or as normally directed. This is the supposition in cases of $\mathrm{BD}$ and in some cases of acute zonal occult outer retinopathy (AZOOR) in which the EOG is severely affected. ${ }^{34}$

In order to gain a better understanding of the underlying pathology, Singh et al. generated an induced pluripotent stem cell (iPS) model for BD. ${ }^{35}$ Initially, RPE cells derived from patients with BD showed no differences from control iPS-RPE cells, however after exposure to a stressor, BD iPS-RPE cells demonstrated reduced phagocytosis and accumulation of autofluorescent material. ${ }^{35}$ Although in vivo all RPE cells should be equally susceptible to the effects of mutation, it may be that only RPE cells responsible for maintaining the foveal photoreceptors are sufficiently stressed to demonstrate functional abnormalities, as subretinal deposit rarely accumulates outside the macula in BD. As both 
the amplitude of the light rise and the presence of maculopathy can be individually and independently expressed, the underlying mechanisms may be divergent. The processes responsible for generating the EOG are less likely to involve the phagocytic pathway but may be calcium dependant, as BD iPS-RPE cells exhibit altered endoplasmic reticulum (ER) dependent calcium currents when compared to control cells. ${ }^{35,36}$ Indeed it has been suggested that the RPE acting alone can respond to photic stimulation, generating an increase in inositol triphosphate (IP3), resulting in calcium release from the ER into the cytosol, followed by transport of chloride ions across the basolateral RPE membrane. ${ }^{37}$ Restoration of intracellular calcium levels is thought to require both Ltype calcium channels as well as BEST1, acting to transport chloride as a counter ion. ${ }^{38}$

Recently it has been suggested that there is a dynamic relationship in the distance between the photoreceptor outer segment (POS) and the inner surface of the RPE, so called "outer segment equivalent length" (OSEL), such that in light the two are closely approximated whereas in the dark they lay further apart. ${ }^{39}$ Abramoff et al. suggest that these morphological changes have a similar time course to the EOG changes in light and dark; and that these changes are abolished, and even reversed in patients with BD and an abnormal EOG, where there is chronic OSEL lengthening rather than shortening in response to light. ${ }^{39}$ They suggest that one role of BEST1 is to enable the approximation of the RPE and photoreceptor outer segment thus enhancing the efficiency of phagocytosis. We now propose an alternative hypothesis, that BEST1 facilitates pigment granule migration within the RPE. In the dark-adapted state Johnson et al. have shown that these granules aggregate towards the base of the cell. ${ }^{40}$ Under such conditions OCT measurement of OSEL would represent the distance from the ellipsoid zone to the lower half of the RPE (where the pigment granules reside). In the light the granules disperse, mediated by a calcium dependant mechanism, and now preferentially reside within the apical processes of the RPE. ${ }^{40}$ The OSEL would now be correspondingly shorter, and this shortening would be determined by pigment migration rather than the length of the photoreceptor outer segment. In BD, dysfunctional BEST1 protein within the ER would disrupt normal calcium homeostasis, resulting in less pigment dispersion and reduced 
migration to the apical processes. ${ }^{40}$ Accordingly the OSEL would not be expected to reduce in BD as observed in the original study. ${ }^{39}$

BEST1 function is therefore thought to be critical for multiple but independent cellular processes, probably indirectly mediated by its ability to regulate intracellular calcium levels. The effects of mutation are correspondingly diverse. Firstly, it can reduce light induced chloride efflux at the basolateral surface of the RPE (most likely through TMEM16A calcium gated chloride channels), as mutant BEST1 channels may result in reduced ER calcium stores. If these stores are replenished through a different pathway, chloride efflux may still be supported and an EOG light rise may be generated. Secondly, BEST1 mutation can reduce POS phagocytosis, a process similarly controlled by intracellular calcium. Thirdly, altered pigment granule migration is thought to be calcium dependant, and is worthy of further investigation as a novel endophenotype in BD. The current work now provides clinical evidence that the EOG phenotype is more variable than once thought. As the calcium sensitive chloride channels, phagocytic process and pigment granule migration pathways are all likely to have differential calcium sensitivities, it is perhaps not surprising that patients with BEST1 mutations show such variation in disease expression. 


\section{REFERENCES}

1. Petrukhin $\mathrm{K}$ et al. Identification of the gene responsible for Best macular dystrophy. Nature genetics 1998; 19:241-247.

2. Marquardt A et al. Mutations in a novel gene, VMD2, encoding a protein of unknown properties cause juvenile-onset vitelliform macular dystrophy (Best's disease). Human molecular genetics 1998; 7:1517-1525.

3. Kramer $\mathrm{F}$ et al. Mutations in the VMD2 gene are associated with juvenile-onset vitelliform macular dystrophy (Best disease) and adult vitelliform macular dystrophy but not age-related macular degeneration. European journal of human genetics : EJHG 2000; 8:286-292.

4. Davidson AE et al. Missense mutations in a retinal pigment epithelium protein, bestrophin-1, cause retinitis pigmentosa. American journal of human genetics 2009; 85:581-592.

5. Yardley $\mathrm{J}$ et al. Mutations of VMD2 splicing regulators cause nanophthalmos and autosomal dominant vitreoretinochoroidopathy (ADVIRC). Investigative ophthalmology \& visual science 2004; 45:3683-3689.

6. Burgess $R$ et al. Biallelic mutation of BEST1 causes a distinct retinopathy in humans. American journal of human genetics 2008; 82:19-31.

7. Michaelides $\mathrm{M}$ et al. Evidence of genetic heterogeneity in MRCS (microcornea, rod-cone dystrophy, cataract, and posterior staphyloma) syndrome. American journal of ophthalmology 2006; 141:418-420.

8. Boon CJ et al. The spectrum of ocular phenotypes caused by mutations in the BEST1 gene. Progress in retinal and eye research 2009; 28:187-205.

9. Deutman AF. Electro-oculography in families with vitelliform dystrophy of the fovea. Detection of the carrier state. Archives of ophthalmology (Chicago, III : 1960) 1969; 81:305-316.

10. Hood DC et al. ISCEV standard for clinical multifocal electroretinography (mfERG) (2011 edition). Documenta ophthalmologica Advances in ophthalmology 2012; 124:1-13.

11. Bach $\mathrm{M}$ et al. ISCEV standard for clinical pattern electroretinography (PERG): 2012 update. Documenta ophthalmologica Advances in ophthalmology 2013; 126:1-7.

12. McCulloch DL et al. ISCEV Standard for full-field clinical electroretinography (2015 update). Documenta ophthalmologica Advances in ophthalmology 2015; 130:1-12.

13. Querques $\mathrm{G}$ et al. Functional and clinical data of Best vitelliform macular dystrophy patients with mutations in the BEST1 gene. Molecular vision 2009; 15:2960-2972.

14. Sodi A et al. Ocular phenotypes associated with biallelic mutations in BEST1 in Italian patients. Molecular vision 2011; 17:3078-3087.

15. Kinnick TR et al. Autosomal recessive vitelliform macular dystrophy in a large cohort of vitelliform macular dystrophy patients. Retina 2011; 31:581-595.

16. Wittstrom $\mathrm{E}$ et al. Morphological and functional changes in multifocal vitelliform retinopathy and biallelic mutations in BEST1. Ophthalmic genetics 2011; 32:83-96.

17. Seddon JM et al. Assessment of mutations in the Best macular dystrophy (VMD2) gene in patients with adult-onset foveomacular vitelliform dystrophy, age-related maculopathy, and bull'seye maculopathy. Ophthalmology 2001; 108:2060-2067.

18. ExAC Browser (Beta) | Exome Aggregation Consortium.

19. Caldwell GM et al. Bestrophin gene mutations in patients with Best vitelliform macular dystrophy. Genomics 1999; 58:98-101.

20. Pollack K, Kreuz FR and Pillunat LE. [Best's disease with normal EOG. Case report of familial macular dystrophy]. Der Ophthalmologe : Zeitschrift der Deutschen Ophthalmologischen Gesellschaft 2005; 102:891-894.

21. Wabbels B et al. Genotype-phenotype correlation and longitudinal course in ten families with Best vitelliform macular dystrophy. Graefe's archive for clinical and experimental ophthalmology = Albrecht von Graefes Archiv fur klinische und experimentelle Ophthalmologie 2006; 244:1453-1466. 
22. Testa $\mathrm{F}$ et al. A normal electro-oculography in a family affected by best disease with a novel spontaneous mutation of the BEST1 gene. The British journal of ophthalmology 2008; 92:1467-1470. 23. Low $\mathrm{S}$ et al. Autosomal dominant Best disease with an unusual electrooculographic light rise and risk of angle-closure glaucoma: a clinical and molecular genetic study. Molecular vision 2011;

17:2272-2282.

24. Pomares E et al. Nonsense-mediated decay as the molecular cause for autosomal recessive bestrophinopathy in two unrelated families. Investigative ophthalmology \& visual science 2012; 53:532-537.

25. Leiden Open Variation: Bestrophin 1(BEST1).

26. Seddon JM et al. Dietary fat and risk for advanced age-related macular degeneration. Archives of ophthalmology (Chicago, III : 1960) 2001; 119:1191-1199.

27. Yu J et al. Biochemical analysis of a common human polymorphism associated with agerelated macular degeneration. Biochemistry 2007; 46:8451-8461.

28. Weleber RG. Fast and slow oscillations of the electro-oculogram in Best's macular dystrophy and retinitis pigmentosa. Archives of ophthalmology 1989; 107:530-537.

29. Lobes LA. The electro-oculogram in human retinal detachment. The British journal of ophthalmology 1978; 62:223-226.

30. Arden GB and Wolf JE. Differential effects of light and alcohol on the electro-oculographic responses of patients with age-related macular disease. Investigative ophthalmology \& visual science 2003; 44:3226-3232.

31. Harding GF et al. Electro-oculography, electroretinography, visual evoked potentials, and multifocal electroretinography in patients with vigabatrin-attributed visual field constriction. Epilepsia 2000; 41:1420-1431.

32. Arndt CF et al. Retinal electrophysiological results in patients receiving lamotrigine monotherapy. Epilepsia 2005; 46:1055-1060.

33. Kawasaki $\mathrm{K}$ et al. Acetazolamide-induced changes of the membrane potentials of the retinal pigment epithelial cell. Documenta ophthalmologica Advances in ophthalmology 1986; 63:375-381.

34. Francis PJ et al. Acute zonal occult outer retinopathy: towards a set of diagnostic criteria. The British journal of ophthalmology 2005; 89:70-73.

35. Singh $\mathrm{R}$ et al. iPS cell modeling of Best disease: insights into the pathophysiology of an inherited macular degeneration. Human molecular genetics 2013; 22:593-607.

36. Constable PA. A perspective on the mechanism of the light-rise of the electrooculogram. Investigative ophthalmology \& visual science 2014; 55:2669-2673.

37. Rodriguez de Turco EB, Gordon WC and Bazan NG. Light stimulates in vivo inositol lipid turnover in frog retinal pigment epithelial cells at the onset of shedding and phagocytosis of photoreceptor membranes. Experimental eye research 1992; 55:719-725.

38. Gomez NM, Tamm ER and Straubeta O. Role of bestrophin-1 in store-operated calcium entry in retinal pigment epithelium. Pflugers Archiv : European journal of physiology 2013; 465:481-495.

39. Abramoff MD et al. Human photoreceptor outer segments shorten during light adaptation. Investigative ophthalmology \& visual science 2013; 54:3721-3728.

40. Johnson AS and Garcia DM. Carbachol-mediated pigment granule dispersion in retinal pigment epithelium requires Ca2+ and calcineurin. BMC cell biology 2007; 8:53. 


\section{LEGENDS}

Figure 1. Multimodal imaging in Patient 3 with molecularly proven autosomal recessive betrophinopathy (ARB). (A) Near infra-red reflectance imaging demonstrating central macular lesions. (B) $50^{\circ} 488 \mathrm{~nm}$ fundus autofluorescence imaging highlights the absence of extra-macular disease, which is usually a typical feature of ARB, and macular lesions at different stages of disease. (C) Optical coherence tomography shows well-defined outer retinal atrophy in the right eye and an earlier stage of disease characterised by subretinal fluid in the left eye. Another feature of ARB, intraretinal fluid is notably absent.

Figure 2. Multimodal imaging of Patient 6 over two years showing features typically associated with Best Disease. (A) Near infra-red reflectance imaging identifies focal central macular lesions in both eyes. (B) 488nm fundus autofluorescence imaging highlights an initial uniform increase in signal at the right macula, which later becomes more heterogeneously distributed, possibly under the influence of gravity, and a lack of physiological autofluorescence from the left eye. (C) Optical coherence tomography is helpful in demonstrating the subretinal location of the vitelliform material in the right eye, and the extent of outer retinal atrophy in the left eye.

Table 1. A summary of all known BEST1 variants which are associated with a higher than expected Arden ratio. The data is ordered based on the position of the mutant residue. The current work (Patients 1, 3, 4 and 5) is presented alongside previously reported studies. In all cases testing incorporated the relevant ISCEV standards of the time. 